\title{
Prevalence of normoalbuminuric renal insufficiency and associated clinical factors in adult onset diabetes
}

\author{
Polwatta Liyanage Gayani Chandima Liyanage ${ }^{1 *}$, Sarath Lekamwasam², Thilak Priyantha Weerarathna ${ }^{2}$ and \\ Dodanduwa Waduge Shyamal Yashodara Srikantha ${ }^{1}$
}

\begin{abstract}
Background: Microalbuminuria signifies the onset of diabetic nephropathy, but normoalbuminuric patients with diabetes who have a low Glomerular Filtration Rate (GFR) are not uncommon. The purpose of the study was to estimate the prevalence of such patients and to assess the clinical correlates.

Methods: Cross-sectional study included patients with diabetes attending medical clinics at Teaching Hospital Galle. Diagnosis of albuminuria was made if urinary albumin excretion was $>30 \mathrm{mg} / \mathrm{g}$ of creatinine in two out of three samples. Patients were stratified into chronic kidney disease stages according to the estimated GFR (eGFR) calculated by Modification of Diet in Renal Disease (MDRD).

Results: Mean (SD) age and duration of the disease of 456 (348 females) patients with diabetes were 60 (12) years and 10 (4) years. Sixty (13.2\%) patients had low eGFR and 26.7\% of them had normoalbuminuria. In the total sample, the proportion of patients with low eGFR and normoalbuminuria was 16 (3.5\%). Among the patients with normoalbuminuria and low eGFR, $12.5 \%$ had retinopathy and none had any form neuropathy. When age, duration of disease, systolic and diastolic blood pressures, smoking, glycaemic control, presence of hypertension and ischaemic heart disease were included in binary logistic regression model, only age was found to be significant different $(O R=1.1, P=0.03)$.

Conclusion: A considerable proportion of adult diabetics are normoalbuminuric despite low eGFR. This limits the role of microalbuminuria as a screening tool to detect the onset of diabetic nephropathy. These patients do not exhibit distinct clinical features that facilitate identification of them using clinical information.
\end{abstract}

Keywords: Albuminuria, Chronic kidney disease, Diabetes

\section{Background}

Of the long standing complications of diabetes, diabetic nephropathy (DN) has gained attention of clinicians and researches due to its progression to Chronic Kidney Disease (CKD) and End Stage Renal Disease (ESRD). Approximately $30 \%$ of the patients on renal replacement therapy have diabetes [1].

Chronic hyperglycemia and resulting advanced glycated end products in diabetes are the two most likely causative factors of DN [2]. Natural history of DN

\footnotetext{
* Correspondence: gayanicl@yahoo.com

${ }^{1}$ Department of Pharmacology ,Faculty of Medicine, University of Ruhuna, Galle, Sri Lanka

Full list of author information is available at the end of the article
}

comprises of five stages; glomerular hypertrophy and hyperfiltration, renal structural changes without microalbuminuria, microalbuminuria with preserved renal function, significant proteinuria with progressive renal impairment and finally the development of ESRD [3]. Most accurate information on severity of DN is provided by histological evaluation of the affected kidneys.

The development of microalbuminuria is considered to be the first biochemical marker of diabetic nephropathy [4] while persistent albuminuria indicates overt nephropathy [5]. Renal insufficiency is characterized and staged reliably by calculating estimated glomerular filtration rate (eGFR) from an abbreviated equation from the Modification of Diet in Renal Disease (MDRD) study. 
CKD is diagnosed when e GFR is less than $60 \mathrm{ml} / \mathrm{min} /$ $1.73 \mathrm{~m}^{2}$ for at least 3 months duration [6]. It has been found that there is a good correlation between reduction of eGFR and degree of albuminuria in a proportion of patients with DN [7].

Although it is believed that DN begins with microalbuminuria and progresses to overt albuminuria before renal insufficiency occurs, studies have shown that a significant proportion of patients do not follow this set pattern and have low e GFR without albuminuria. This entity is called normoalbuminuric renal impairment (NARI) and in patients with type 2 diabetes it has a distinct clinical picture and is not clearly associated with poor glycaemic control [8]. The causation and pathophysiology of this distinct type of renal involvement in diabetes, however, has not been studied in detail in South Asian region. This cross-sectional study was aimed to estimate the prevalence of normoalbuminuric renal impairment and to study its clinical associations among patients with diabetes attending a tertiary care hospital in Southern Sri Lanka.

\section{Methods}

This cross -sectional study included randomly selected patients more than 20 years of age with diabetes attending medical clinics at Teaching Hospital, Galle (THG). Pregnant or lactating women and patients with acute or chronic infections were excluded. Patients with diseases that can potentially influence renal function or albuminuria such as systemic lupus erythematosus, urosepsis, and nephrotic syndrome were also excluded.

Patients were identified using patient registries maintained in four medical clinics and out of them 600 patients were selected randomly and screened at the Department of Medicine on a selected date. After explaining the procedure an informed written consent was obtained from all patients (Fig. 1).

In the subjects selected, morning urine samples were collected and urine dipstick test was done to exclude ongoing urinary tract infections. (Urine collection was postponed if patient had fever, urinary symptoms suggestive of a urinary tract infection or menstruation). Only the urine samples negative for nitrates were stored at $-80{ }^{\circ} \mathrm{C}$ for urine albumin analysis. Presence of albuminuria was tested by turbidimetric method [9]. Creatinine content in urine samples was measured by Jaffe reaction [10]. Each patient underwent testing of urine three times during the course of 3 months and categorization of microalbuminuria was confirmed if two out of three urine samples were positive for albumin ( $>30 \mathrm{mg}$ albumin/g of creatinine). Those who had albuminuria $>300 \mathrm{mg}$ albumin/g of creatinine were diagnosed as having macroalbuminuria.
Selected patients with diabetes $(\mathrm{n}=600)$

Patients with recurrent urinary tract

infections and haematuria $(n=16)$

Symptomatic heart failure $(\mathrm{n}=3)$

Non diabetic renal disease $(n=3)$

Patients did not complete the urinary tests $(\mathrm{n}=96)$

Patients with diabetes completed the study $(\mathrm{n}=482 \mathrm{t}$

Fig. 1 Flow chart of the study participants

Identification of the presence of chronic kidney disease and its stage among the study population was done by $\mathrm{e}$ GFR values obtained using MDRD formula [6]. The degree of glycemic control was assessed by $\mathrm{HbA}_{1} \mathrm{C}$ level done by HPLC method [11].

A total of 600 patients with diabetes attending medical clinics at Teaching Hospital Karapitiya, Sri Lanka were selected randomly. Patients with type 2 diabetes who had recurrent urinary tract infections and haematuria $(n=16)$, symptomatic heart failure $(n=3)$, non-diabetic renal disease $(n=3)$ and those who did not complete the urinary tests $(n=96)$ were excluded from the study.

Anthropometric measurements such as weight, height, waist and hip circumferences were measured using standard scales and techniques. Blood pressure was measured in the sitting position in the right arm with a digital sphygmomanometer after $15 \mathrm{~min}$ of resting. Average of two readings which were taken $5 \mathrm{~min}$ apart was considered as the final blood pressure reading. Systolic blood pressure response to standing and diastolic blood pressure response to sustained handgrip were measured. Ankle Brachial Pressure Index (ABPI) was measured using mini doppler. It was classified as low $\leq 0.9$, normal $0.9-1.3$ and high $>1.3$.

The validated Sinhala version of Rose Angina Questionnaire (RAQ) was administered to the subjects. This questionnaire was administered by a medical officer who had a prior knowledge on the rules applicable to filling the questionnaire. Angina and possible infarction were defined according to the World Health Organization (WHO) guidelines given with the original RAQ.

Retinopathy was detected by direct ophthalmoscopy using a Heine ophthalmoscope in a darkened room. In doubtful and difficult situations, the help of the consultant ophthalmologist were sought.

Diabetic Neuropathy Symptom (DNS) and Diabetic Neuropathy Examination (DNE) scores were obtained 
according to the guidelines. Score of more than 1 in DNS and more than 3 in DNE were considered having diabetic peripheral neuropathy. Semmes Weinstein Monofilament (SW-MF) $10 \mathrm{~g}$ was used to detect neuropathy according to the standard "yes/no" method of administration.

Further, we made an extensive search in the history and clinical examination to detect non-diabetic renal disease. In addition previous case records were examined. None of the patients included in the current study had evidence previous acute kidney injury or non-diabetic renal disease.

\section{Statistical analysis}

All statistical analyses were performed using the Statistical Package for the Social Sciences. One way Analysis Of Variance (ANOVA) and unpaired t-test were used to compare continuous variables and Chi-square test was performed to compare categorical variables in different groups. Logistic regression analyses were performed to detect the determinants and associations of albuminuria and low e GFR. $P<0.05$ was considered statistically significant.

\section{Results}

The mean (SD) age of the study sample $(n=482)$ was 61 (11) years and $75 \%$ of them $(n=360)$ were females. Of the patients with diabetes studied, 286 (60.9\%) had microalbuminuria and 24 (5.1\%) had macroalbuminuria. The prevalence of diabetic nephropathy defined according to albuminuria (both micro and macro) was $66 \%$.

Regarding the treatment, 369 (76\%) of patients were on Angiotensin Converting Enzyme Inhibitor (ACEI) or Angiotensin Receptor Blocker (ARB). Percentage of ACEI or ARB treated patients in normoalbuminuric, microalbuminuric and macroalbuminuric patients were 68.8, 78.3 and $81.1 \%(P=0.57)$ respectively.

Descriptive data of the patients participated in the study are shown in Table 1.

One hundred and seventy four (42.9\%) patients had eGFR $<60 \mathrm{ml} / \mathrm{min}$ and of them $43(43 / 174,24.7 \%)$ were normoalbuminuric. In the total sample, the proportion of patients with low eGFR and normoalbuminuria was 43 (10.8\%) (Table 2).

Comparison of clinical characteristics among patients with diabetes when considered both albuminuria and eGFR is shown in Table 3.

Table 4 shows a comparison of clinical characteristics in relation to nephropathy status according to albuminuria among patients with diabetes. Patients with albuminuria had a longer duration of diabetes when compared with normoalbuminuric patients $(P<0.05)$. Systolic blood pressure, age and poor glycaemic control were significantly higher among patients with
Table 1 Descriptive data of patients with diabetes included in the study $(n=482)$

\begin{tabular}{ll}
\hline Factor & Percentage \\
\hline Age (years) $^{\text {a }}$ & $60.7(10.5)$ \\
Gender (male) & $24.9 \%$ \\
Retinopathy & $21.4 \%$ \\
Angina according to the RAQ & $23 \%$ \\
MI according to the RAQ & $3.5 \%$ \\
ABPI & \\
Lowest to 0.9 & $11.9 \%$ \\
0.9 to 1.3 & $86.2 \%$ \\
$>1.3$ & $1.9 \%$ \\
Hypertension & $68.7 \%$ \\
Neuropathy & \\
DNS & $19.5 \%$ \\
DNE & $13.1 \%$ \\
Monofilament & $39 \%$ \\
Biothesiometer & $3.5 \%$ \\
Postural drop & $23.4 \%$ \\
Handgrip & \\
Abnormal & $31.7 \%$
\end{tabular}

${ }^{\mathrm{a} G i v e n ~ m e a n ~(S D) ~}$

$M I$ (Myocardial infarction), RAQ (Rose angina questionnaire), ABPI (Ankle brachial pressure index), DNS (Diabetic Neuropathy Symptom score), DNE (Diabetic Neuropathy Examination score)

albuminuria when compared with normoalbuminuric patients $(P<0.05)$. Smoking, Body Mass Index (BMI), Diastolic Blood Pressure (DBP), gender and Waist Hip Ratio (WHR), however, were not significantly different between patients with albuminuria and normoalbuminuria.

Regression analysis revealed that poor glycemic control and duration of diabetes were significant associations of albuminuria in patients with diabetes (Table 5). Although there were many variables associated with albuminuria, the regression model retained only poor glycaemic control and disease duration as significant associations of albuminuria.

Table 2 Number of patients with type 2 diabetes classified by CKD stages and albuminuria stages

\begin{tabular}{lll}
\hline & $\begin{array}{l}\text { Normoalbuminura } \\
N=134\end{array}$ & $\begin{array}{l}\text { Albuminuria } \\
N=262\end{array}$ \\
\hline $\begin{array}{l}\text { CKD 1-2 }(n=228) \\
\text { eGFR } \geq 60 \mathrm{ml} / \mathrm{min}\end{array}$ & 91 & 137 \\
CKD 3 $(n=164)$ & 43 & 121 \\
eGFR 30-59 ml/min & & \\
CKD $4(n=3)$ & 0 & 3 \\
eGFR 15-29 ml/min & & 1 \\
CKD 5 $(n=1)$ & 0 & \\
eGFR $<15 \mathrm{ml} / \mathrm{min}$ & & \\
\hline
\end{tabular}

CKD (Chronic kidney disease) 
Table 3 Comparison of clinical characteristics among patients with diabetes when considered both albuminuria and eGFR

\begin{tabular}{llllll}
\hline & $\begin{array}{l}\text { Normoalbuminuric } \\
\& \text { eGFR }>60 \\
n=92\end{array}$ & $\begin{array}{l}\text { Normoalbuminuric } \\
\& \text { eGFR }<60 \\
n=43\end{array}$ & $\begin{array}{l}\text { Albuminuric } \\
\& \text { eGFR }>60 \\
n=138\end{array}$ & $\begin{array}{l}\text { Albuminuric } \\
\& \text { eGFR }<60 \\
n=127\end{array}$ \\
\hline Retinopathy & $3(3.3 \%)$ & $3(7.0 \%)$ & $43(32.3 \%)$ & $40(33.1 \%)$ & $<$ value \\
Neuropathy & $21(23.1 \%)$ & $17(40.5 \%)$ & $57(41.6 \%)$ & $64(50.4 \%)$ & 0.001 \\
Low ABPI & $6(6.5 \%)$ & $6(14.0 \%)$ & $18(13.1 \%)$ & $18(14.4 \%)$ & 0.56 \\
Hypertension & $55(59.8 \%)$ & $33(76.7 \%)$ & $89(64.5 \%)$ & $37(76.4 \%)$ & 0.03 \\
Rose angina & $17(23 \%)$ & $9(30 \%)$ & $32(25.6 \%)$ & $35(29.2 \%)$ & 0.77 \\
\hline
\end{tabular}

Values are number (\%)

$A B P I$ (ankle brachial pressure index)

For the next analyses, nephropathy was defined based on the e GFR regardless of albuminuria. In these patients comparison of clinical characteristics with the CKD stages is shown in Table 6.

Regression analysis revealed that glycemic control and duration of diabetes were significant associations of albuminuria in patients with diabetes (Table 5). Although there were many variables associated with albuminuria, the regression model retained only poor glycaemic control and disease duration as significant associations of albuminuria.

In the regression analysis, smoking and age were the significant determinants of low e GFR (Table 7).

\section{Discussion}

Results of this study reveal that among patients with renal insufficiency (e GFR $<60 \mathrm{ml} / \mathrm{min} / 1.73 \mathrm{~m}^{2}$ ) $26.7 \%$ were normoalbuminuric. According to several cross-sectional and cohort studies, it has been proven that the prevalence of NARI is not an infrequent finding among patients with diabetes [11]. The prevalence of NARI varies from 14 to $57 \%$ in cross- sectional studies carried out in various countries [11]. Our finding on prevalence of NARI is consistent with the most of the previous studies conducted in other countries such as in Australia and the USA where prevalence of NARI is $23.2 \%$ and $27 \%$ respectively [12].
Further, So et al. in 2006 reported prevalence of NARI as low as $14 \%$ in 4421 patients he studied [13].However, another study from Japan has reported a higher prevalence of NARI with, $51.8 \%$ of patient with low eGFR $(<60 \%)$ were normoalbuminuric [14].

The finding that NARI was less strongly associated with other microvascular complications of diabetes was also previously reported. A study from Italy reported that $76.5 \%$ of patients with NARI had no diabetic retinopathy [8]. It has been reported that $75.6 \%$ patients with NARI to be free of other microvascular complication [4]. Furthermore, the same authors reported that $63.4 \%$ of them to have no diabetic nephropathy or neuropathy. This is also consistent with our findings in which $96.7 \%$ of patients with NARI had no diabetic retinopathy and $76.9 \%$ had no neuropathy.

According to the data, the prevalence of albuminuria among total sample was 66\% (95\% CI: 61.7 to 70.2 ) with microalbuminuria was $60.9 \%(n=286)$ while macroalbuminuria prevalence was $5.1 \%(n=24)$.

Among total sample, the prevalence of low eGFR $(<60 \%)$ was $42.9 \%(n=174)$ and age and smoking were the factors related to low eGFR. Other microvascular complication namely, retinopathy and neuropathy were associated with albuminuria but not with low eGFR.

Table 4 Comparison of clinical characteristics between patients with and without albuminuria

\begin{tabular}{llll}
\hline Variable & Normoalbuminuria & Albuminuria & $P$ value \\
\hline Number (\%) & $160(34 \%)$ & $310(66 \%)$ & $62(10)$ \\
Age (years) & $58(11)$ & $79(25.6)$ & $<0.001$ \\
Proportion of males & $36(22.5 \%)$ & $11.2(4.6)$ & 0.45 \\
Duration of diabetes (years) & $7.5(2.7)$ & $40(12.9 \%)$ & $<0.001$ \\
Proportion of Smokers & $17(10.6 \%)$ & $23.7(3.5)$ & 0.47 \\
BMI $\left(\mathrm{kg} / \mathrm{m}^{2}\right)$ & $23.7(3.9)$ & $0.88(0.07)$ & 0.84 \\
WHR & $0.87(0.07)$ & $135.7(18.9)$ & 0.4 \\
SBP $(\mathrm{mmHg})$ & $128.2(18.2)$ & $75.9(10.4)$ & $<0.001$ \\
Mean DBP $(\mathrm{mmHg})$ & $75.3(9.5)$ & $166(70 \%)$ & 0.53 \\
Proportion of poor glycaemic control & $9(7.3 \%)$ & & $<0.001$ \\
\hline
\end{tabular}

Data are mean (SD) or $\mathrm{n}(\%)$

$B M I$ (body mass index), WHR (waist hip ratio), SBP (systolic blood pressure), DBP (diastolic blood pressure) 
Table 5 Association between clinical risk factors and albuminuria (either micro or macro)

\begin{tabular}{lll}
\hline Clinical risk factor & Odds ratio $(95 \% \mathrm{Cl})$ & $P$ value \\
\hline Age & $0.99(0.96$ to 1.03$)$ & 0.87 \\
Smoking & $1.93(0.57$ to 6.52$)$ & 0.29 \\
Duration of diabetes & $1.35(1.21$ to 1.51$)$ & $<0.001$ \\
SBP & $1.01(0.99$ to 1.03$)$ & 0.32 \\
DBP & $0.99(0.95$ to 1.034$)$ & 0.74 \\
Poor glycaemic control & 31.44 (13.82 to 71.52$)$ & $<0.001$ \\
\hline SBP (systolic blood pressure), DBP (diastolic blood pressure) &
\end{tabular}

It has been found that normoalbuminuric renal insufficiency is associated with several characteristics other than its association with low prevalence of microvascular complications of diabetes. They include female predominance, lower HbA1c, shorter duration of diabetes and lower prevalence of hypertension [15].

One may consider normoalbuminuric renal insufficiency as the forerunner of microalbuminuric renal insufficiency. In support of this, An J et al. found the prevalence of normoalbuminuric renal insufficiency to decrease with the increased duration of diabetes and advanced stage of retinopathy [15]. It is possible that some patients develop renal insufficiency with no albuminuria initially and then progress to microalbuminuria renal insufficiency as the disease progresses. This may question the use of microalbuminuria as the biochemical hallmark of diabetic nephropathy in clinical practice.

Our data were obtained from a hospital based sample of adult patients with type 2 diabetes, showed a higher prevalence of albuminuria. Although few studies have reported similar figures of albuminuria, most of the studies, both hospital based and population based, have shown
Table 7 Association between clinical risk factors and reduced eGFR (eGFR < 60)

\begin{tabular}{lll}
\hline Clinical risk factor & Odds ratio $(95 \% \mathrm{Cl})$ & $P$ value \\
\hline Age & $1.14(1.1$ to 1.18$)$ & $<0.001$ \\
Smoking & $5.21(1.84$ to 14.7$)$ & 0.002 \\
Duration of diabetes & $0.99(0.93$ to 1.06$)$ & 0.86 \\
SBP & $0.99(0.97$ to 1.01$)$ & 0.35 \\
DBP & $1.02(0.98$ to 1.05$)$ & 0.32 \\
Glycaemic control & 1.1 (0.61 to 1.98$)$ & 0.68 \\
\hline SBP (systolic blood pressure), DBP (diastolic blood pressure) &
\end{tabular}

lower prevalence of albuminuria. A population-based study from India which reported $29.1 \%$ prevalence of albuminuria, but the mean age of the patients was 52 years, about 9 years younger than our study sample [16]. This could be due to several reasons. These include variations in study population, study design and definition of the outcome variable. Compared to other studies our subjects were older and had longer duration of the disease. Higher mean age of the patients in our study could be a reason for the high prevalence of microalbuminuria since albuminuria increases with age. Older patients are likely to have a disease for a longer period, hence more likely to have developed long term complications of diabetes. Further, Unnikrishnan in his study considered presence of diabetic retinopathy in to the definition of diabetic nephropathy [16]. This might also be a reason for getting a less prevalence of albuminuria compared to our study.

Higher prevalence of other types of kidney disease such as chronic kidney disease of unknown origin $(\mathrm{CKDu})$ in the general population in our country may also have contributed to the seemingly high prevalence of albuminuria in this study sample.

Table 6 Comparison of clinical characteristics among patients with diabetes in relation to CKD stages

\begin{tabular}{|c|c|c|c|}
\hline & \multicolumn{2}{|l|}{ CKD stages } & \multirow[t]{2}{*}{$P$ value } \\
\hline & $\begin{array}{l}\text { Stage } 1 \& 2 \\
\text { e GFR }>60\end{array}$ & $\begin{array}{l}\text { Stage } 3,4 \& 5 \\
\text { e GFR }<60\end{array}$ & \\
\hline Number (\%) & $231(57 \%)$ & $174(43 \%)$ & \\
\hline Age (years) & $56(10)$ & $67(8)$ & $<0.001$ \\
\hline Proportion of males & $60(26)$ & $40(23)$ & 0.28 \\
\hline Duration of diabetes (years) & $9(4)$ & $11(4)$ & 0.001 \\
\hline Proportion Smokers & $36(16)$ & $13(7)$ & 0.009 \\
\hline BMI $\left(\mathrm{kg} / \mathrm{m}^{2}\right)$ & $24.7(3.5)$ & $22.3(3.4)$ & $<0.001$ \\
\hline WHR & $0.88(0.06)$ & $0.86(0.07)$ & 0.04 \\
\hline $\mathrm{SBP}(\mathrm{mmHg})$ & $132(17)$ & $136(20)$ & 0.03 \\
\hline $\mathrm{DBP}(\mathrm{mmHg})$ & $76(10)$ & $76(10)$ & 0.96 \\
\hline Proportion of poor glycaemic control (HbA1c > 7.5\%) & $86(47)$ & $58(48 \%)$ & 0.46 \\
\hline
\end{tabular}

Data are means (SD) or $\mathrm{n}(\%)$

$C K D$ (Chronic kidney disease), BMI (body mass index), WHR (waist hip ratio), SBP (systolic blood pressure), DBP (diastolic blood pressure) 


\section{Conclusion}

A considerable proportion of patients with diabetes are normoalbuminuric despite low eGFR. This limits the role of microalbuminuria as a screening tool to detect the onset of diabetic nephropathy. These patients do not exhibit distinct clinical features that facilitate identification of them using clinical information.

\begin{abstract}
Abbreviations
ABPI: Ankle Brachial Pressure Index; ACEl: Angiotensin Converting Enzyme Inhibitor; ANOVA: Analysis of Variance; ARB: Angiotensin Receptor Blocker; BMl: Body Mass Index; CKD: Chronic Kidney Disease; CKDu: Chronic kidney disease of unknown origin; DBP: Diastolic Blood Pressure; DN: Diabetic nephropathy; DNE: Diabetic Neuropathy Examination; DNS: Diabetic Neuropathy Symptom; eGFR: estimated GFR; ESRD: End Stage Renal Disease; GFR: Glomerular Filtration Rate; HPLC: High performance liquid chromatography; MDRD: Modification of Diet in Renal Disease; MI: Myocardial infarction; NARI: Normoalbuminuric renal impairment; RAQ: Rose Angina Questionnaire; SW-MF: Semmes Weinstein Monofilament; THG: Teaching Hospital, Galle; WHO: World Health Organization; WHR: Waist Hip Ratio
\end{abstract}

\section{Acknowledgements}

The authors are indebted to the participants of the study.

\section{Funding}

This study was funded by University of Ruhuna Research Grant and University Grants Commission Grant in Sri Lanka. The study sponsor had no role in study design, data collection, data analysis, data interpretation or writing the report.

\section{Availability of data and materials}

The datasets used and analyzed during the current study are available from the corresponding author on reasonable request.

\section{Authors' contributions}

GL conceived the idea, planned the study, collected patient information, analyzed and interpreted data, drafted the paper and took part in revising and finalizing the manuscript. SL contributed for designing the study, supervised the study, analyzed and interpreted data, edited the manuscript and approved the final version. TW contributed for designing the study, supervised the study, edited the manuscript and approved final version. YS contributed in revising and finalizing the manuscript. All authors are in agreement with the content of the manuscript.

\section{Ethics approval and consent to participate}

Ethical approval for this study was obtained from ethical review committee of Faculty of Medicine, University of Ruhuna, Galle. Informed written consent was obtained from all individuals prior to data collection. Participants were informed of their rights to withdraw from the study at any stage.

\section{Consent for publication}

Not applicable.

\section{Competing interests}

The authors declare that they have no competing interests.

\section{Publisher's Note}

Springer Nature remains neutral with regard to jurisdictional claims in published maps and institutional affiliations.

\section{Author details}

'Department of Pharmacology ,Faculty of Medicine, University of Ruhuna, Galle, Sri Lanka. ${ }^{2}$ Department of Medicine, Faculty of Medicine, University of Ruhuna, Galle, Sri Lanka.
Received: 26 October 2017 Accepted: 3 August 2018

Published online: 13 August 2018

\section{References}

1. Locatelli F. Renal replacement therapy in patients with diabetes and end-stage renal disease. J Am Soc Nephrol. 2004;15:25S-29.

2. Peppa M, Vlassara $\mathrm{H}$. Advanced glycation end products and diabetic complications: a general overview. HORMONES. 2005:4:28-37.

3. Adler A.I., Stevens R.J., Manley S.E., Bilous R.W., Cull C.A., Holman R.R. (UKPDS GROUP). Development and progression of nephropathy in Type 2 diabetes: The United Kingdom Prospective Diabetes Study (UKPDS 64) Kidney Int. 2003; 63:225-232.

4. Yokoyama $\mathrm{H}$, Sone $\mathrm{H}$, Oishi M, Kawai $\mathrm{K}$, Fukumoto $\mathrm{Y}$, Kobayashi M. Prevalence of albuminuria and renal insufficiency and associated clinical factors in type 2 diabetes: the Japan diabetes clinical data management study (JDDM15). Nephrology Dialysis Transplantation. 2008;24:1212-9.

5. Parving HH, Mauer M, Ritz E. Diabetic nephropathy. In: Brenner B, editor. Brenner and Rector's the kidney. 8th ed. volume 2. Saunders. Philadelphia: PA, USA; 2007. p. 1265-98.

6. KDOQI. Clinical practice guidelines and clinical practice recommendations for diabetes and chronic kidney disease. Am J Kidney Dis. 2007;49:S12-S154.

7. Saha T, Bhattarai A, Batra H, Banerjee M, Misra P, Ambade V. Correlation of microalbuminuria with estimated GFR (eGFR) by Cockcroft-gault and MDRD formula in type 2 diabetics and hypertensives. Indian J Clin Biochem. 2014;30:271-4.

8. Penno G, Solini A, Bonora E, Fondelli C, Orsi E, Zerbini G, Trevisan R, Vedovato M, Gruden G, Cavalot F, et al. Clinical significance of nonalbuminuric renal impairment in type 2 diabetes. J Hypertens. 2011;29:1802-9.

9. Redon J. Measurement of microalbuminuria - what the nephrologist should know. Nephrol Dial Transplant. 2006;21:573-6.

10. Delanghe J, Speeckaert M. Creatinine determination according to Jaffe-what does it stand for? Clinical kidney journal. 2011;4:83-86. A highperformance liquid chromatography method for hemoglobin A1c. Diabetes. 1978;27:102-7.

11. Esteban P, Piero R, Carl E, Drazenka P, Manuel P, Josep M, Radovan H, Manuela A, Aiko P. Non-proteinuric pathways in loss of renal function in patients with type 2 diabetes. Lancet Diabetes Endocrinol. 2015;3:382-91.

12. Maclsaac RJ, Tsalamandris C, Panagiotopoulos S, et al. Nonalbuminuric renal insufficiency in type 2 diabetes. Diabetes Care. 2004;27:195-200.

13. So WY, Kong AP, Ma RC. Glomerular filtration rate, cardiorenal end points, and all-cause mortality in type 2 diabetic patients. Diabetes Care. 2006;29:2046-52.

14. Yokoyama H, Kawai K, Kobayashi M. Japan diabetes clinical data management study Group. Microalbuminuria is common in Japanese type 2 diabetic patients: a nationwide survey from the Japan diabetes clinical data management study Group (JDDM 10). Diabetes Care. 2007;30:989-92.

15. An J, Cho Y, Yu H, Jang H, Park K, Kim S, et al. The clinical characteristics of Normoalbuminuric renal insufficiency in Korean type 2 diabetic patients: a possible early stage renal complication. J Korean Med Sci. 2009;24(Suppl 1):S75

16. Unnikrishnan R, Rema M, Pradeepa R, Deepa M, Shanthirani C, Deepa R, et al. Prevalence and risk factors of diabetic nephropathy in an urban south Indian population: the Chennai urban rural epidemiology study (CURES 45). Diabetes Care. 2007:30(8):2019-24. 